Bundesgesundheitsbl 2009 $\cdot 52: 557-570$

DOI 10.1007/s00103-009-0843-3

Online publiziert: 4. April 2009

(c) Springer Medizin Verlag 2009

\author{
B.-M. Kurth · C. Lange · P. Kamtsiuris · H. Hölling \\ Abteilung für Epidemiologie und Gesundheitsberichterstattung, \\ Robert Koch-Institut, Berlin
}

\title{
Gesundheitsmonitoring am Robert Koch-Institut
}

\author{
Sachstand und Perspektiven
}

\section{Aufgaben des Robert Koch- Instituts als Public-Health- Institut und die Rolle der Gesundheitsberichterstattung}

$\mathrm{Zu}$ den neuen Herausforderungen an die Gesunderhaltung der Bevölkerung und damit an das Gesundheitssystem in Deutschland zählt die wachsende Lebenserwartung der Bevölkerung, die einhergeht mit sinkenden Geburtenraten, was insgesamt zu einer Alterung der Gesellschaft führt. Immer mehr Menschen leben mit chronischen Krankheiten, ohne an ihnen zu versterben. Dies ist auf Fortschritte in der medizinischen Versorgung zurückzuführen, steigert aber die Kosten für das Gesundheitswesen weiter. Gleichzeitig nimmt die Verbreitung von Risikofaktoren für Herz-Kreislauf-Krankheiten wie Übergewicht und körperliche Inaktivität in der Bevölkerung zu. Das Auftreten neuer Infektionskrankheiten als Folge von Globalisierung und Klimawandel sowie das Wiedererstarken von bereits für besiegt geglaubten Infektionskrankheiten durch Multiresistenzen und sich partiell verschlechternden Impfschutz erfordern ebenfalls neue Strategien für den Gesundheitsschutz. Um diesen Herausforderungen angemessen begegnen zu können, wurde und wird das Robert Koch-Institut (RKI) im Rahmen des Projekts „RKI 2010" als modernes Public-Health-Institut gestärkt und neu gestaltet.

Die zentralen Aufgaben des Instituts im Sinne eines Public-Health-Instituts bestehen im
- frühzeitigenErkennenvongesundheitsrelevanten Problemen in der Allgemeinbevölkerung und in Risikogruppen,

- unabhängigen Bewerten von Entwicklungen, Problemlagen und Risiken auf Basis erhobener Daten, - Entwickeln von Maßnahmen zur Gesundheitsförderung und Krankheitsverhütung (Handeln) [1].

Für die epidemiologisch arbeitenden Abteilungen des RKI bedeutet „Erkennen“ die kontinuierliche Beobachtung der Entwicklung von Krankheitshäufigkeiten und Krankheitsrisiken in der Gesellschaft unter Berücksichtigung gesundheitsförderlicher und gesundheitsgefährdender Lebensbedingungen.

Unabhängiges „Bewerten“ von Entwicklungen, Problemlagen und Risiken beinhaltet die wissenschaftliche und von Interessen Dritter freie Auswertung und Bewertung der vorhandenen Informationen und Daten, um prioritäre Gesundheitsprobleme identifizieren zu können. Mit der Gesundheitsberichterstattung des Bundes (GBE), die seit 1999 in der Verantwortung des RKI liegt, wird seit nunmehr zehn Jahren dieser Aufgabe nachgegangen. In Kooperation mit dem Statistischen Bundesamt liefert die GBE daten- und indikatorengestützte ${ }^{1}$ Beschreibungen und Ana-

\footnotetext{
1 Der Begriff "Indikator" wird in diesem Text als Synonym verwendet für eine epidemiologische Kenngröße (zum Beispiel Häufigkeit von Arztkontakten, Anteil der Raucher, Menge des täglichen Alkoholkonsums bezogen auf eine definierte Bevölkerungsgruppe), die Auskunft gibt über die Quantität eines Gesundheitsfaktors.
}

lysen zu allen Bereichen des Gesundheitswesens: gesundheitliche Lage, Gesundheitsprobleme und Krankheiten, Gesundheitsverhalten und -gefährdungen, Leistungen und Inanspruchnahme, Ausgaben und Kosten der Gesundheitsversorgung, Ressourcen und Rahmenbedingungen. Die hierzu erforderliche Datenbasis wird regelmäßig aktualisiert und über das Informationssystem der GBE des Bundes zur Verfügung gestellt (http://www.gbe-bund.de).

Da Daten aus amtlichen Statistiken, Register- und Prozessdaten die Themenfelder der GBE nur partiell abdecken, sind Daten aus Gesundheitssurveys unverzichtbar, um bestehende Informationslücken zu füllen. Bevölkerungsbezogene Gesundheitssurveys liefern insbesondere Daten zu Gesundheitsstatus, Gesundheitsverhalten, Krankheiten sowie Versorgung der Bevölkerung und ermöglichen, diese mit den Informationen zu Lebensbedingungen zu verknüpfen. Anders als Prozessdaten des Gesundheitssystems ermöglichen sie auch Aussagen zu denjenigen Personen, die das gesundheitliche System nicht in Anspruch nehmen. Die Daten aus den Gesundheitssurveys des RKI finden vielfältig Eingang in die Gesundheitsberichterstattung des Bundes [2]: Sowohl in dem im Jahr 2007 erschienenen Gesundheitsbericht für Deutschland als auch in den monatlich erscheinenden GBE-Themenheften wird für wesentliche Aussagen auf Daten des Bundes-Gesundheitssurveys 1998, des Kinder- und Jugendgesundheitssurveys KiGGS sowie der telefonischen Gesundheitssurveys zurückgegriffen. Auch bei der Festlegung von Ge- 


\begin{tabular}{|c|c|c|c|c|c|c|c|c|}
\hline Jahr & Methode & Region & Alter & $\begin{array}{l}\text { Stichpro- } \\
\text { bengröße }\end{array}$ & Männer & Frauen & $\begin{array}{l}\text { Ausschöp- } \\
\text { fungsquote }\end{array}$ & Finanzierung \\
\hline \multicolumn{9}{|c|}{ Nationale Untersuchungssurveys } \\
\hline $1984-86$ & $\begin{array}{l}\text { Schriftliche Befragung + Untersu- } \\
\text { chung }\end{array}$ & Westdeutschland & $25-69$ & 4790 & 2417 & 2373 & $66,0 \%$ & BMBF \\
\hline $1987-88$ & $\begin{array}{l}\text { Schriftliche Befragung + Untersu- } \\
\text { chung }\end{array}$ & Westdeutschland & $25-69$ & 5335 & 2649 & 2686 & $71,4 \%$ & $\mathrm{BMBF}$ \\
\hline 1990-91 & $\begin{array}{l}\text { Schriftliche Befragung + Untersu- } \\
\text { chung }\end{array}$ & Westdeutschland & $25-69$ & 5311 & 2623 & 2688 & $69,0 \%$ & BMBF \\
\hline \multicolumn{9}{|c|}{ Gesundheitssurvey Ost } \\
\hline 1991-92 & $\begin{array}{l}\text { Schriftliche Befragung + Untersu- } \\
\text { chung }\end{array}$ & Ostdeutschland & $18-79$ & 2617 & 1223 & 1394 & $70,2 \%$ & BMG \\
\hline \multicolumn{9}{|c|}{ Bundes-Gesundheitssurvey } \\
\hline 1998 & $\begin{array}{l}\text { Schriftliche Befragung + Untersu- } \\
\text { chung }\end{array}$ & Gesamtdeutschland & $18-79$ & 7124 & 3450 & 3674 & $61,4 \%$ & BMG/BMBF/RKI \\
\hline \multicolumn{9}{|c|}{ Telefonische Gesundheitssurveys } \\
\hline 2003 & Telefonische Befragung & Gesamtdeutschland & $18+$ & 8318 & 3872 & 4446 & $59,2 \%$ & BMG/RKI \\
\hline 2004 & Telefonische Befragung & Gesamtdeutschland & $18+$ & 7341 & 3376 & 3965 & $56,1 \%$ & BMG/RKI \\
\hline 2005 & Telefonische Befragung & Gesamtdeutschland & $18+$ & 7692 & 3517 & 4151 & $57,3 \%$ & BMG/RKI \\
\hline 2006 & Telefonische Befragung & Gesamtdeutschland & $18+$ & 5542 & 2476 & 3066 & $56,1 \%$ & BMG/RKI \\
\hline \multicolumn{9}{|c|}{ Kinder- und Jugendgesundheitssurvey } \\
\hline $2003-06$ & $\begin{array}{l}\text { Schriftliche } \\
\text { Befragung + Un- } \\
\text { tersuchung }\end{array}$ & Gesamtdeutschland & $0-17$ & 17.641 & 8985 & 8656 & $66,6 \%$ & BMG/BMBF/RKI \\
\hline
\end{tabular}

sundheitszielen auf Bundesebene [3] wurden zur Beschreibung der Ausgangsbasis die Ergebnisse telefonischer Gesundheitssurveys verwendet, die teilweise entsprechende thematische Module enthielten [4, $5,6,7,8,9]$. Daten aus Gesundheitssurveys des RKI fließen auch in andere Berichterstattungssysteme ein, so in die Armutsund Reichtumsberichterstattung [10], die Ernährungsberichterstattung [11] oder die Umweltberichterstattung.

\section{Bisherige Gesundheitssurveys am RKI und daraus abgeleitete Anforderungen an künftige Erhebungen}

Unter dem Titel „Nationale Gesundheitssurveys" wurden bereits in den Jahren 198486 (NUSTo) und 1987-89 (NUST1) sowie 1990-91 (NUST2) repräsentative Stichproben der bundesdeutschen Bevölkerung im Rahmen der Deutschen Herz-Kreislauf-Präventionsstudie (DHP) einer standardisierten Untersuchung und einer umfangreichen Befragung zu gesundheitsrelevanten Themen unterzogen. Schwerpunkt dieser Erhebungen waren Herz-KreislaufErkrankungen und deren Risikofaktoren. Die Surveys sollen Auskunft geben über die Entwicklung von Gesundheitszustand, Gesundheitsverhalten und Gesundheitsrisiken in der sogenannten Normalpopulation, sprich, der Bevölkerung, bei der keinerlei Interventionsversuche im Rahmen der DHP unternommen wurden. Diese drei Gesundheitssurveys wurden dreimal hintereinander mit identischen Instrumenten durchgeführt [12].

Mit weitgehend gleicher Methodik wie bei den DHP-Surveys wurde auch in den neuen Bundesländern 1991-92 ein Gesundheitssurvey (Survey Ost) durchgeführt. Hierbei war es von großem gesundheitspolitischem Interesse, zeitnah nach der Wiedervereinigung beider deutscher Staaten möglicherweise existierende Unterschiede im Gesundheitszustand und Gesundheitsverhalten sowie in der gesundheitlichen Versorgung der Bürger in Ostund Westdeutschland aufzudecken.

Der Bundes-Gesundheitssurvey 1998 wurde dann unter dem breiteren Blickwinkel der Gesundheitsberichterstattung und im Kontext der Einordnung in die Forschungsanliegen des RKI neu konzipiert. Besonderes Augenmerk wurde dabei der Entwicklung der 1991/92 festgestellten Unterschiede im Gesundheitszustand und -verhalten der Ost- und West- deutschen geschenkt (vergleiche [13]). Der Ansatz, zunehmend international verwendete und validierte Erhebungsinstrumente einzusetzen, barg die Chance in sich, mit anderen europäischen, aber auch internationalen Studien kompatibel zu sein.

Die mithilfe der Gesundheitssurveys beantworteten Fragen der Gesundheitspolitik und die Nutzung der Daten für die inzwischen etablierte Gesundheitsberichterstattung des Bundes führten zu einer immer größeren Nachfrage nach aktuellen Informationen. Zur Überbrückung der größeren zeitlichen Abstände zwischen den Gesundheitssurveys mit Untersuchungskomponente, aber auch aus Kosten- und Effektivitätsgründen, wurde am RKI ein Instrumentarium für telefonische Befragungen zu Gesundheitsthemen implementiert [14].

In den Jahren 2003-2006 hat das RKI vier bundesweite telefonische Gesundheitssurveys im Auftrag des Bundesministeriums für Gesundheit (BMG) durchgeführt. Sie hatten einen Umfang von jeweils etwa 7000 Interviews. Ein methodisch ausgerichteter telefonischer Survey zur Erprobung des Einsatzes international etablierter Instrumente am Telefon, beispielsweise zur Erhebung der psychischen 
Gesundheit und des Gesundheitsverhaltens, folgte im Jahr 2007.

Eine Altersgruppe war bis zum Jahr 2003 bei allen Surveyerhebungen des RKI ausgespart geblieben: die o- bis 17-Jährigen. 1998 erhielt das RKI vom BMG den Auftrag, Konzept und Instrumente für einen Kinder- und Jugendgesundheitssurvey zu entwickeln. Aufbauend auf die Erfahrungen der Erwachsenensurveys wurde über mehrere Jahre ein wiederholt begutachtetes und auch über eine Ethikkommission abgesichertes Projekt entwickelt und in einem Pretest getestet [15]. Die Finanzierung des darauf aufbauenden Kinder- und Jugendgesundheitssurveys (KiGGS), der von 2003-2006 insgesamt 17.641 Kinder und Jugendliche sowie deren Eltern Deutschlands in 167 Orten einbezog, wurde gemeinsam durch das BMG, das Bundesministerium für Bildung und Forschung (BMBF) und das RKI sichergestellt (http://www.kiggs.de). In diesem Survey lag erstmals auch die Verantwortung für die Feldarbeit beim RKI, da sich eine externe Vergabe als zu teuer erwies. Die positiven Erfahrungen, die diese Vorgehensweise hinsichtlich Response und Qualität mit sich brachte, führten zu der Entscheidung, künftige Gesundheitserhebungen in RKI-Eigenregie durchzuführen.

Die Kennzahlen aller RKI-Surveys sind in - Tab. 1 zusammengefasst.

\section{Konzept eines Gesundheits- monitorings am RKI}

Wie aus $\bullet$ Tab. 1 ersichtlich, konnte eine regelmäßige Erhebungsperiodik der Surveys nicht eingehalten werden. Die Zeitabstände zwischen den Befragungs- und Untersuchungssurveys waren nicht nur unterschiedlich groß, sondern auch relativ lang, was hauptsächlich finanztechnisch bedingt war. Die jeweilige Finanzierung der Gesundheitssurveys erfolgte ausschließlich über die Einwerbung von Drittmitteln über Projektanträge. Eine Planbarkeit und kontinuierliche Entwicklung der einzelnen Gesundheitssurveys war nicht gegeben. Diese Vorgehensweise wurde ineffizient, zumal eine zunehmend evidenzbasierte Gesundheitspolitik auf regelmäßig erhobene, belastbare Daten sowohl zur Zieldefinition als auch zur Eva-

Bundesgesundheitsbl 2009 · 52:557-570 DOI 10.1007/s00103-009-0843-3

(C) Springer Medizin Verlag 2009

\section{B.-M. Kurth · C. Lange · P. Kamtsiuris · H. Hölling Gesundheitsmonitoring am Robert Koch- Institut. Sachstand und Perspektiven}

\section{Zusammenfassung}

Seit Januar 2008 gibt es am Robert Koch-Institut (RKI) ein Gesundheitsmonitoring, das auf alternierend durchzuführenden Untersuchungs- beziehungsweise Befragungssurveys mit Längsschnittkomponenten beruht und kontinuierlich durch jährliche Querschnittsbefragungen der in Deutschland lebenden Bevölkerung begleitet wird. Das inhaltliche und strukturelle Konzept dieses Monitoringsystems wird erläutert, die aktuell laufenden Surveys werden vorgestellt. Ein Ausblick auf künftige Erhebungen und die Einordnung in den europäischen Kontext verdeutlicht die qualitativ neuen Nutzungsmöglichkeiten der im Längsschnitt und Querschnitt erhobenen Daten für die Gesundheitsberichterstattung, die Gesundheitspolitik und die Gesundheitswissenschaften.

Schlüsselwörter

Gesundheitsmonitoring · Gesundheitssurveys · Längsschnittstudie · Kohorte

\section{Health monitoring at the Robert Koch Institute. Status and perspectives}

\section{Abstract}

Since January 2008, the Robert Koch Institute (RKI) has been administrating a Health Monitoring System, i.e., health examination surveys and health interview surveys with longitudinal components being alternatingly performed. These surveys are continuously accompanied by annual cross-sectional interview surveys of the population living in Germany. The content and structure of the Health Monitoring System is explained and the actual on-going surveys are described in this paper. An overview of future surveys and their integration within the European context demonstrates the new quality in utilizing the data obtained by longitudinal and cross-sectional analyses for health reporting, health policies and health sciences.

\section{Keywords}

Health monitoring · Health surveys · Longitudinal studies · Cohorts 
luation der Prozesse und Ergebnisse zurückgreifen wollte [16].

Aus diesem Grunde wurde durch das RKI unaufgefordert ein Konzept für ein kontinuierliches Gesundheitsmonitoring entwickelt und 2005 im Bundesgesundheitsblatt publiziert [17]. Dabei wurde das Anliegen eines Gesundheitsmonitorings definiert als

- die kontinuierliche Beobachtung von Entwicklungen im Krankheitsgeschehen, im Gesundheits- und Risikoverhalten und im Umgang mit Krankheiten und gesundheitlichen Risiken,

- die Identifizierung von Trends und deren Veränderungen sowie deren Analyse im Verhältnis zu möglichen oder bisher gängigen Interventionen.

Dafür muss ein Gesundheitsmonitoring unter Einbeziehung des Gesamtnetzwerks dokumentierter und nutzbarer gesundheitsorientierter Daten regelmäßig belastbare Informationen zu allen Bereichen des Gesundheitssystems liefern. Die Grundprinzipien des Gesundheitsmonitorings bestehen daher darin, „Vorhandenes zu nutzen“, „Lücken zu identifizieren und zu füllen“ sowie „Informationen zu vernetzen“. In [17] wurde eine Bestandsaufnahme bereits existierender Monitoringkomponenten in Deutschland vorgenommen. Dazu gehören kontinuierlich laufende Erhebungen, wie beispielsweise die Mortalitätsstatistik, die Krebsregistrierung sowie die Surveillance meldepflichtiger Infektionskrankheiten nach Infektionsschutzgesetz. Die hieraus abzuleitenden Indikatoren stehen kontinuierlich zur Verfügung.

Informationen hingegen, die ausschließlich über zeitlich nicht planbare Surveyerhebungen gewonnen werden konnten, standen nicht in regelmäßigen Abständen zur Verfügung. Sie werden jedoch regelmäßig benötigt. Gesundheitssurveys sind gewöhnlich Querschnittserhebungen, die repräsentative Informationen zu einem bestimmten Zeitpunkt für eine Bevölkerungsgruppe liefern. Aussagen zu zeitlichen Abfolgen, UrsacheWirkungs-Beziehungen und individuellen Lebensverläufen lassen sich auf dieser Datengrundlage selbst bei wiederholten Querschnittserhebungen nicht treffen. Daher ist es erstrebenswert, trotz hö- herer Kosten und schwieriger Logistik, Probanden aus Surveys über einen längeren Zeitraum wiederholt zu befragen und zu untersuchen. In Schlussfolgerung daraus und unter Berücksichtigung der Erfahrungen mit der Durchführung von Gesundheitssurveys sah das Konzept für ein Gesundheitsmonitoring des RKI folgende Komponenten vor:

- regelmäßig wiederkehrende, methodisch vergleichbare bevölkerungsrepräsentative Querschnittserhebungen zu subjektiver und objektiver Gesundheit, gesundheitsrelevanten Verhaltensweisen und Einstellungen,

- Durchführung von Interview- und Untersuchungssurveys nach einem festgelegten zeitlichen Turnus und Erhebungsschlüssel,

- Kombination von immer mitgeführten Kernmodulen (zur Sicherung von Trendanalysen) mit variablen Untersuchungsteilen (zum Erhalt der Flexibilität),

- Durchführung von Längsschnittbeobachtungen in Untergruppen.

Diese Monitoringkomponenten sind dabei so konzipiert, dass im zeitlichen Verlauf folgende Vergleiche möglich werden:

Querschnittsvergleiche. Unterschiedliche Probandengruppen können zu jedem Erhebungszeitpunkt hinsichtlich aller erhobenen Merkmale verglichen werden. Der Vergleich über die Zeit ermöglicht Aussagen zu Gesundheitstrends in der Gesamtbevölkerung bzw. in Bevölkerungsgruppen.

Kohortenvergleiche. Studienteilnehmer verschiedener Geburtsjahrgänge werden, über die verschiedenen Erhebungszeitpunkte hinweg, beobachtet und miteinander verglichen. Es können sowohl Trend- als auch Verlaufsanalysen durchgeführt werden.

Lebenslaufvergleiche. Studienteilnehmer, die einer bestimmten Kohorte (zum Beispiel einem bestimmten Geburtsjahrgang) angehören, werden über die einzelnen Erhebungszeitpunkte verfolgt, sodass deren Entwicklungen über mehrere Zeitpunkte beschrieben und analysiert, Übergänge und Verläufe feststellt und damit auch kausale Zusammenhänge aufgedeckt werden können.

\section{Die Umsetzung des Konzepts für ein Gesundheitsmo- nitorings am RKI}

Mit einer umfassenden Begründung der Sinnhaftigkeit und Effizienz eines solchen Gesundheitsmonitorings im Hinblick auf die Belange der Gesundheitspolitik, der Gesundheitsberichterstattung, aber auch der Gesundheitswissenschaften trat das RKI im Jahr 2005 an das BMG heran, um über die Finanzierbarkeit zu verhandeln. Im Ergebnis konstruktiver inhaltlicher Diskussionen wurde das Monitoringkonzept weiter konkretisiert. Bereits im Jahr 2007 ermöglichte eine Mittelzuweisung des BMG an das RKI den Start in die Umsetzungsphase. Als im Rahmen des Projekts „RKI 2010“ ab 2008 Personal und Sachmittel für die Realisierung des „RKI-Gesundheitsmonitorings“ zur Verfügung gestellt wurden, war das RKI-Gesundheitsmonitoring nicht mehr nur „Vision“, sondern nunmehr „Mission" und nahm sehr konkrete Formen an: In konsequenter Umsetzung des theoretischen Monitoringkonzepts [18] unter Nutzung bereits am RKI durchgeführter Gesundheitssurveys setzt sich das RKIGesundheitsmonitoring nunmehr konkret aus drei Komponenten zusammen (- Abb. 1):

GEDA: Gesundheit in Deutschland aktuell. Jährlich replizierte Querschnittsbefragungen der erwachsenen Wohnbevölkerung im Alter von über 18 Jahren. Die Stichprobengröße von ca. 20.00o Teilnehmerinnen und Teilnehmern erlaubt dabei tief gegliederte Zusammenhangsanalysen von sozialer Lage, Gesundheitszustand, Gesundheitsverhalten und Inanspruchnahme gesundheitlicher Versorgung. Eine Repräsentativität auf regionaler Ebene ist für die größeren Bundesländer gewährleistet. Diese Daten erlauben Trendanalysen sowie eine rasche Bestandsaufnahme von Änderungen des Gesundheitszustands oder des Gesundheitsverhaltens.

DEGS: Studie zur Gesundheit Erwachsener in Deutschland. Periodische Durch- 
führung eines Befragungs- und Untersuchungssurveys bei Erwachsenen zur Erhebung objektiver Messwerte (Größe und Gewicht, Blutdruck, körperliche Funktionsfähigkeit, ärztliche Untersuchung, Abnahme von Blut- und Urinproben zur Bestimmung vielfältiger Biomarker) und zur vertieften Bearbeitung spezifischer Gesundheits- oder Krankheitsprobleme. Dabei soll durch Wiedereinbeziehung von Probanden vorangegangener Erhebungen eine Längsschnittkomponente integriert sein, um Lebensverläufe und kausale Abfolgen beschreiben zu können. Aufgrund der aufwendigen und standardisierten Befragungs-, Mess- und Erhebungsverfahren (Laboruntersuchungen), die einen personellen Aufwand durch den Einsatz von Feldteams und den Aufbau temporärer Studienzentren erfordern, können diese Untersuchungssurveys nur in größeren zeitlichen Abständen durchgeführt werden. Zwischenzeitlich sollen daher reine Befragungssurveys durchgeführt werden.

KiGGS: Studie zur Gesundheit von Kindern und Jugendlichen in Deutschland. Fortführung des KiGGS [19] als Kohorte. Es ist geplant, die gesamte Gruppe der in KiGGS einbezogenen Kinder und Jugendlichen ab 2009 über drei Jahre hinweg erneut (telefonisch) zu befragen und zusätzlich neu rekrutierte o- bis 6-jährige Kinder in die Erhebung einzubeziehen (KIGGS-Welle 1), um aktuelle Daten über die Gesundheit der nachwachsenden Generation zu erhalten. Nach Beendigung der telefonischen Befragungen ist geplant, ab 2012 einen erneuten Untersuchungssurvey mit der „KiGGS-Kohorte“ durchzuführen.

$\mathrm{Da}$ alle drei Monitoringkomponenten vom RKI gleichzeitig durchgeführt werden, sind aus Gründen der Logistik und der Finanzierbarkeit die alternierenden Abfolgen von Untersuchungs- und Befragungssurveys bei KiGGS und DEGS so konzipiert, dass jeweils nur ein Untersuchungssurvey läuft, entweder in der Gruppe der Kinder und Jugendlichen oder bei den Erwachsenen.

Nachfolgend werden die drei Surveykomponenten des Gesundheitsmonitorings und der Stand der Realisierung in den Jahren 2008/2009 beschrieben. Vorangestellt sei, dass alle Erhebungen dem

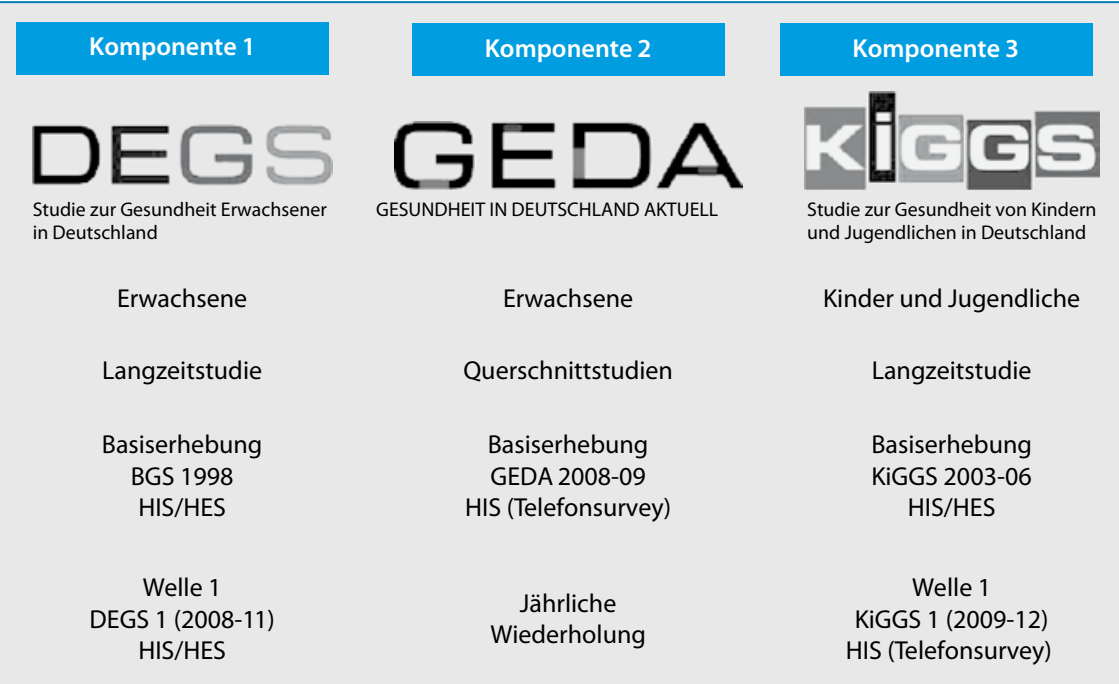

Abb. $1 \Delta$ Komponenten des Gesundheitsmonitorings

Bundesbeauftragten für Datenschutz vor$\mathrm{ab}$ vorgelegt und dessen Anmerkungen berücksichtigt wurden. Positive Voten von Ethikkommissionen liegen ebenfalls vor.

\section{DEGS: Studie zur Gesundheit Erwachsener in Deutschland}

DEGS ist in der ersten Welle ein Untersuchungs- und Interviewsurvey, der anders als reine Interviewsurveys den persönlichen Kontakt mit dem Probanden und körperliche Untersuchungen voraussetzt. Solche Surveys sind sehr aufwendig und logistisch schwieriger, weil die Studienteilnehmer gewöhnlich in Studienzentren eingeladen werden und die gesamte Studie sehr personalintensiv und damit teuer ist. Aber dieser Aufwand macht die dabei gesammelten Daten besonders wertvoll: Während viele Informationen zur Gesundheit und ihren Bedingungen im Rahmen von Befragungen zu erheben sind, sind für die Beantwortung einer Reihe von Fragestellungen zusätzlich objektivierbare Messwerte unabdingbar. Dies betrifft beispielsweise die Messung von Größe und Gewicht zur Berechnung des Body-Maß-Indexes, die Messung des Blutdrucks zur Bestimmung der Häufigkeit von (unerkannter) Hypertonie, die Untersuchung des Blutzuckerspiegels beziehungsweise Nüchternblutzuckerbestimmungen zur Abschätzung des (unerkannten) Diabetes mellitus sowie die Bestimmung von Biomarkern wie Serumlipidspiegeln oder Markern für Herz-Kreislauf-Krankheiten, Nierenkrankheiten, In- fektionskrankheiten und für andere akute oder chronische Krankheiten. Die Kombination aus Angaben der Probanden, objektiven Messungen und Untersuchungen sowie die Analyse von biologischen Proben ermöglicht es, sehr belastbare und mehrfach abgesicherte Aussagen zum Gesundheitszustand der Probanden zu treffen.

Zielpopulation des DEGS „Studie zur Gesundheit Erwachsener in Deutschland“ sind die in der Bundesrepublik Deutschland aktuell lebenden und in den Einwohnermelderegistern mit Hauptwohnsitz gemeldeten Erwachsenen im Alter von 1879 Jahren. Auch ausländische Mitbürger, deren Hauptwohnsitz in Deutschland liegt, werden in den Survey einbezogen.

Mit DEGS realisiert das RKI erstmalig eine längsschnittliche Komponente in einem Survey: Die wieder auffindbaren Probanden des Bundes-Gesundheitssurveys 1998 (BGS 98) [13] werden in den aktuellen Survey erneut einbezogen. Fast alle Teilnehmer des BGS 98 hatten ihre Bereitschaft zur Teilnahme an einer neuen Befragung und Untersuchung bekundet. Die ältesten Teilnehmer des BGS 98 haben mittlerweile ein Alter von 89 Jahren erreicht, auch sie werden nach Möglichkeit in die Studie einbezogen. Darüber hinaus wird die Stichprobe um neue Probanden erweitert, um die aktuelle Bevölkerungsstruktur abzubilden (so muss beispielsweise die Altersgruppe 18-27 Jahre komplett durch neu gezogene Probanden abgedeckt werden, die höheren Altersgruppen müssen durch neu gezogene 
Aus den Herausgeberinstituten

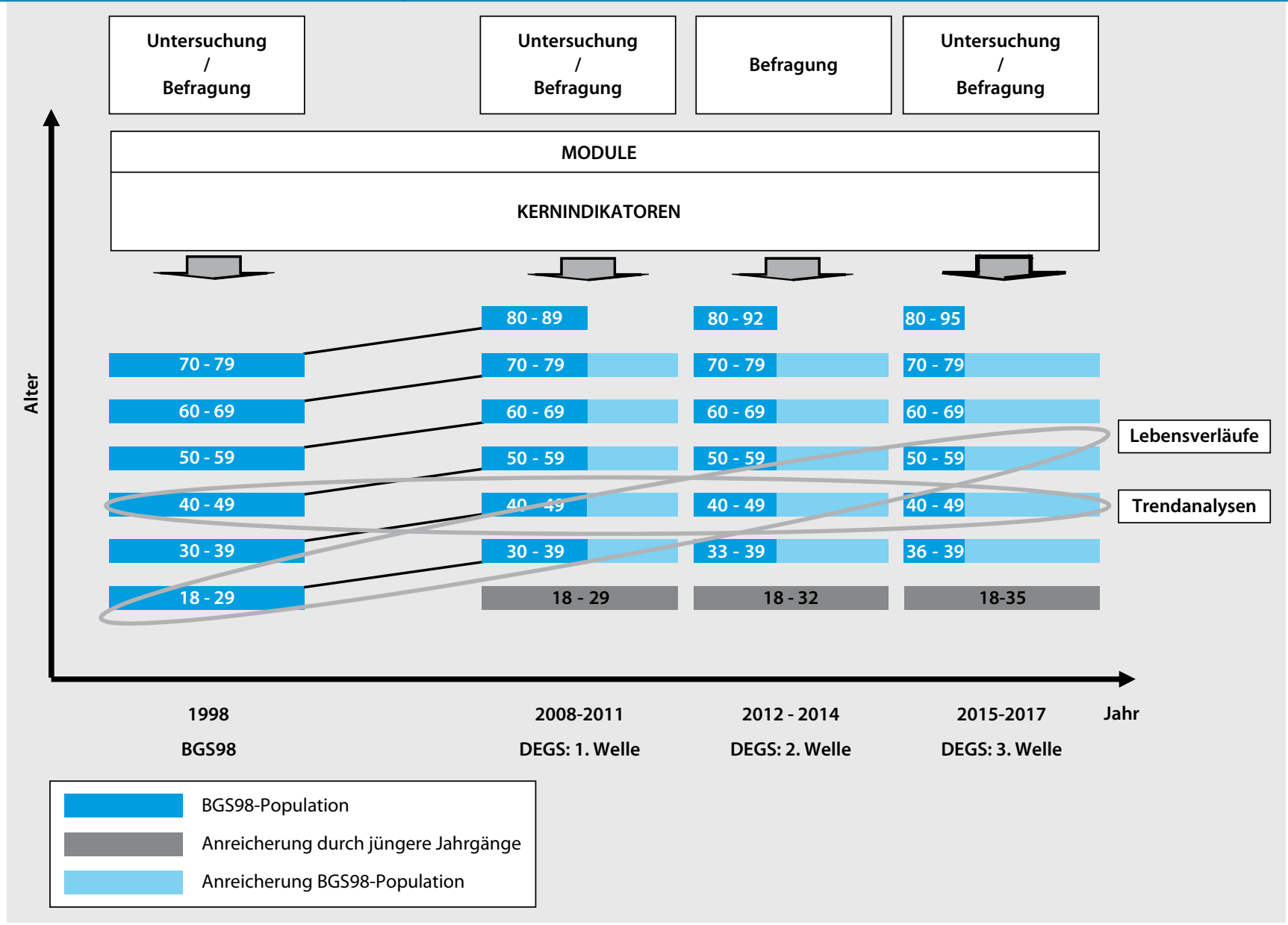

Abb. $2 \Delta$ Schematischer Aufbau des Längsschnittdesigns der DEGS-Studie

Probanden aufgefüllt werden). Die DEGSProbanden sollen im Zeitverlauf in mehreren Erhebungswellen abwechselnd befragt beziehungsweise befragt und untersucht werden (• Abb. 2).

Die 120 Orte (Sample Points) des BGS 98 werden beibehalten und durch 60 neue Points ergänzt. Sehr große Gemeinden (zum Beispiel Berlin und Hamburg) sind mit mehreren Sample Points in der Stichprobe vertreten. Jedem Sample Point entspricht ein eigener Untersuchungszeitraum, das heißt, diese großen Städte werden im Verlauf des Surveys mehrfach angefahren.

Am 25. November 2008 hat das RKI mit den Datenerhebungen für die bundesweite „Studie zur Gesundheit Erwachsener in Deutschland“ (DEGS) begonnen. Die Datenerhebungen werden von zwei RKI-Feldteams durchgeführt. Jedes Team besteht aus einer Ärztin/einem Arzt, einer Untersucherin, einer MTA und einer/m Zentrumsinterviewer/in. Die beiden Feldteams wurden im November
2008 umfassend geschult. Die Teams sind in den einzelnen Orten sechs Tage (Montag bis Samstag) zu Gast. In jeder Untersuchungswoche werden etwa 42-45 Teilnehmerinnen und Teilnehmer untersucht.

Die Arbeit der Teams wird durch eine interne (RKI) und eine externe Qualitätskontrolle (BIPS, Bremer Institut für Prävention und Sozialforschung) ständig überwacht und qualitätsgesichert.

Die Beschreibung des Gesamtprojekts, einschließlich der Inhalte der einzelnen Untersuchungs- und Befragungsteile erscheint demnächst in der Reihe „Beiträge zur Gesundheitsberichterstattung “ des Bundes [20].

\section{GEDA: Gesundheit in Deutschland aktuell}

In Ergänzung und Verschränkung zu DEGS, dessen Durchführung erhebliche Ressourcen beansprucht und dessen Feldphase über drei Jahre hinweg verläuft, werden in der Studie „Gesundheit in Deutschland aktuell“ (GEDA) Probanden $z u$ ihrer gesundheitlichen Situation im Allgemeinen und zu spezifischen aktuellen gesundheitsrelevanten Themen telefonisch befragt [21]. GEDA stellt eine Fortführung der seit 2003 am RKI etablierten telefonischen Gesundheitssurveys dar [22]. Diese Befragungen sollen in jährlichen Wellen der Gesundheitsberichterstattung und der Gesundheitspolitik zeitnah aktuelle Daten zur gesundheitlichen Lage der Bevölkerung zur Verfügung stellen. Die kostengünstige und schnelle Art der Datengewinnung ermöglicht es, flexibel auf neue Erkenntnisse, Anforderungen und Fragen reagieren zu können. Im internationalen Raum sind telefonische Gesundheitsbefragungen schon wesentlich länger etabliert: Das Behavioral Risk Factor Surveillance System in den USA beispielsweise besteht seit 1984 (http://www. cdc.gov/brfss/) und liefert wesentliche Erkenntnisse zur Entwicklung des Gesundheitszustands und des Gesundheitsverhaltens in allen Bundesstaaten der USA. 
Bei GEDA werden in jeder Welle jeweils unabhängige Stichproben der erwachsenen Wohnbevölkerung über 18 Jahren telefonisch befragt. Zur Durchführung der Interviews wurde am RKI ein eigenes CATI-Labor (Computer-AssistedTelephone-Interview) mit 40 Arbeitsplätzen aufgebaut. Über 500 besonders geschulte Interviewer befragen die Teilnehmerinnen und Teilnehmer an sechs Tagen der Woche (Montag-Samstag) zwischen 10 und 20 Uhr. Die Stichprobengröße von rund 20.000 Teilnehmerinnen und Teilnehmern erlaubt regionale Analysen, die für größere Bundesländer repräsentativ sind. Landesspezifische Aufstockungen der Stichprobe können auch für kleinere Länder repräsentative Aussagen ermöglichen; bislang haben das Saarland und Brandenburg von dieser Option Gebrauch gemacht. Beide Länder finanzieren eine landesspezifische Aufstockung um jeweils etwa 2000 Interviews, die repräsentative Aussagen differenziert nach Geschlecht und drei Altersgruppen für das jeweilige Bundesland ermöglichen. Brandenburg wird sich an einer Welle, das Saarland über drei Erhebungswellen hinweg beteiligen. Stichprobenbasis stellt eine nach dem Gabler-HäderVerfahren gezogene Zufallsstichprobe von Telefonnummern dar [23]. Die Grundgesamtheit dieses Auswahlrahmens sind alle in Privathaushalten lebenden Erwachsenen, die über einen Festnetzanschluss verfügen. In methodischen Subprojekten sollen Möglichkeiten der Aufrechterhaltung und weiteren Erhöhung der Teilnahmebereitschaft, der Einsatz weiterer Erhebungsverfahren (schriftlich-postalisch oder über das Internet) sowie der mögliche Wechsel zu einem anderen Stichprobenrahmen untersucht werden. Ähnliche methodische Weiterentwicklungen werden auch im Rahmen des Behavioral Risk Factor Surveillance Systems verfolgt [24].

\section{KiGGS: Studie zur Gesundheit von Kindern und Jugendlichen in Deutschland}

Der KiGGS als erste, bundesweit repräsentative Querschnittsuntersuchung endete nach dreijähriger Feldarbeit im Mai 2006. Erstmalig konnten viele offene Fragen zur Gesundheit und zum Gesund- heitsverhalten der Kinder und Jugendlichen in Deutschland beantwortet werden. Mit jeder gefundenen Antwort entstanden aber auch Folgefragen, wie etwa

- Welche Bedeutung haben bekannte

Risikofaktoren für Herz-Kreislauf-Er-

krankungen im Kindes- und Jugend-

alter? Sind sie veränderbar, sind sie behandlungsbedürftig, und mit welcher Wahrscheinlichkeit führen sie im späteren Leben zu Erkrankungen?

- Wie lange bleiben von Allergien oder Neurodermitis betroffene Kinder krank? Ist zu befürchten, dass neurodermitiskranke Kinder in ihrem späteren Leben ein Asthma bronchiale entwickeln?

- Wie viele Kinder mit psychischen Auffälligkeiten werden tatsächlich krank und nehmen psychologische/ psychotherapeutische Hilfe in Anspruch?

- Wie bewältigen Kinder und Heranwachsende eine Benachteiligung durch Armut oder Migrantenstatus?

- Welche Faktoren sind mit einem Risiko für die Entwicklung von Übergewicht verbunden? Bleiben übergewichtige Kinder übergewichtig? Wie viele dicke Kinder werden dicke Erwachsene? Welche Einflussfaktoren spielen dabei eine Rolle?

Diese Fragen sind nur dann zu beantworten, wenn die Teilnehmer des Kinder- und Jugendgesundheitssurveys weiter beobachtet werden können.

Bereits im Zuge der Konzeption der Querschnitterhebung gab es Vorstellungen, die KiGGS-Kohorte im Längsschnitt weiter zu beobachten und zu begleiten. Dieses Vorhaben wurde in die Gesamtkonzeption eines umfassenden Gesundheitsmonitorings integriert. Der geplante Beobachtungszeitraum beläuft sich vorerst auf etwa 15 Jahre, und die Datenerhebung erfolgt abwechselnd über reine Befragungs- beziehungsweise kombinierte Befragungs- und Untersuchungssurveys. Ziel ist es, die gesundheitliche Entwicklung der Kinder und Jugendlichen bis ins Erwachsenenalter hinein zu verfolgen.

- Abb. 3 stellt exemplarisch die zugrunde gelegte Variante des Längsschnittdesigns bis zur dritten Wiederholungs- welle dar. Dabei wird über jede Welle die ursprüngliche Population des Kinderund Jugendgesundheitssurveys begleitet. Gleichzeitig ist eine Anreicherung zu jedem Erhebungszeitpunkt durch jüngere Jahrgänge vorgesehen, um Kohortenvergleiche zu jedem Erhebungszeitpunkt zu gewährleisten.

Die Feldarbeit zum ersten telefonischen Kinder- und Jugendgesundheitssurvey (KIGGS-Welle) beginnt im Juni 2009. Dazu werden jährlich ca. 8000 Interviews mit Eltern und Kindern beziehungsweise Jugendlichen der KIGGS-Basiserhebung (jetzt 6-23 Jahre) sowie mit Neubefragten (o-6 Jahre) durchgeführt. Eine Vortestung von Feldzugang, Befragungsinstrument und technischer Umsetzung erfolgte in zwei methodischen Vorstudien, die im Februar 2007 mit 318 Probanden und im Juni 2008 mit 154 Probanden durchgeführt wurde.

Die Probanden, die zum Zeitpunkt der Basiserhebung von KiGGS o-17 Jahre alt waren, sind inzwischen 6-23 Jahre alt. Diese Probanden sollen erneut eingeladen werden (Wiederholungsbefragung). Da die Anschriften trotz erfolgter Adresspflege inzwischen ihre Gültigkeit verloren haben können, müssen gegebenenfalls Adressrecherchen durchgeführt werden, wenn die Einladungen nicht mehr zustellbar sind.

Für Probanden des Altersbereichs o6 Jahre erfolgt eine komplette Neuziehung, da dieser Altersbereich ansonsten nicht mehr mit Probanden besetzt wäre. Auch hier werden die zuständigen Einwohnermeldeämter angeschrieben.

Sollte sich im Verlauf der Erhebung zeigen, dass die Teilnehmerzahlen bei den 6- bis 17-Jährigen in bestimmten $\mathrm{Al}$ tersgruppen nicht mehr ausreichen, wird geprüft werden, ob die Stichprobe aufgestockt werden muss, um statistisch gesicherte und bevölkerungsrepräsentative Aussagen treffen zu können.

Einbezogen werden die 167 Sample Points der Basiserhebung. Neue Orte werden nicht hinzugezogen. Probanden, von denen bekannt ist, dass sie generell an keiner Folgeerhebung teilnehmen wollen (zum Beispiel bei Basiserhebungen weitere Kontaktierung auf Einwilligung verweigert) oder können (zum Beispiel Sterbefälle), werden aus dem Einladungsver- 


\section{Aus den Herausgeberinstituten}

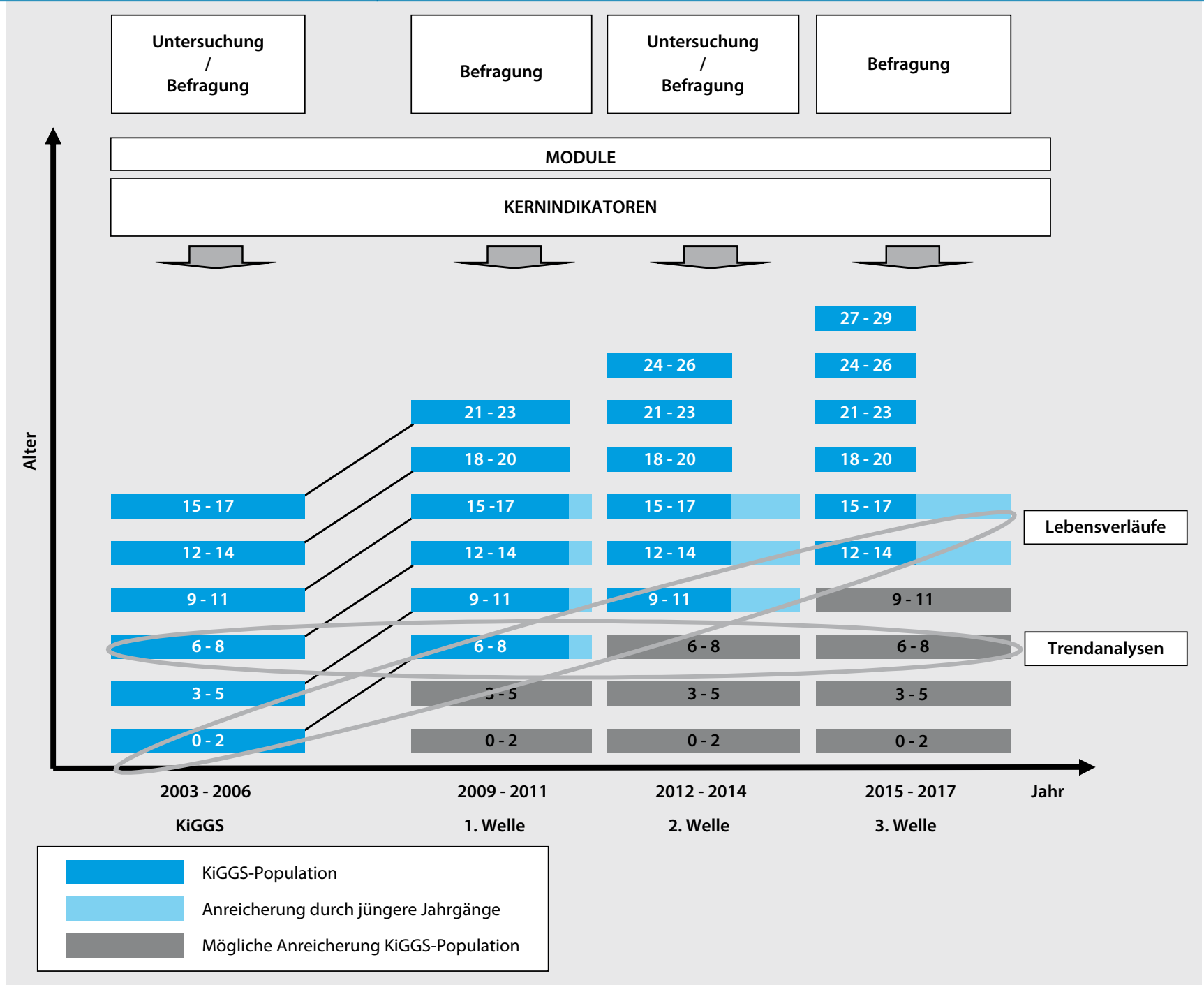

Abb. $3 \Delta$ Schematischer Aufbau des Kohortenansatzes der KiGGS-Studie

fahren ausgeschlossen und als „generelle Ausfälle" deklariert.

\section{Das Gesundheitsmonitoring des RKI: Themenauswahl}

Auch wenn die thematischen Schwerpunkte der Surveyerhebungen bereits grob definiert werden konnten, überschreiten dennoch die Informationsbedarfe und die daraus resultierenden Wünsche schnell den zeitlichen, finanziellen oder organisatorischen Rahmen eines Surveys. Es ist daher unabdingbar, Wünschenswertes von Notwendigem zu unterscheiden und so weit wie möglich objektive Maßstäbe an die Auswahl der inhaltlichen Schwerpunkte anzulegen.
Im Rahmen der Konzeption des bundesweiten Gesundheitsmonitorings wurde auf der Basis bestehender und bewährter Modelle ein spezifisch auf Gesundheitssurveys adaptiertes Priorisierungskonzept erarbeitet. Es fußt auf den Hauptkriterien „Angemessenheit", „Relevanz“, „Messbarkeit“" ,Machbarkeit" und (potenzielle) „Nutzbarkeit“ der Daten.

Angemessenheit: Grundsätzliche Angemessenheit des Themas, keine ethischen oder moralischen Bedenken; Vermeidung von Doppelerhebungen;

Relevanz: Verbreitung, Schweregrad (gesundheitliche Folgen), internationale Anforderungen an Daten zu dem Thema, volkswirtschaftliche Relevanz, Beeinflussbarkeit (zum Beispiel durch Prävention), zukünftige Trendentwicklung;
Messbarkeit: Vorhandensein (international) standardisierter Erhebungsinstrumente; valide Norm- oder Referenzwerte zugänglich;

Machbarkeit: Akzeptanz in der Öffentlichkeit gegeben; Vorhandensein der erforderlichen Ressourcen (Effizienz) und entsprechender technischer Ausstattung;

Nutzbarkeit: Nutzbarkeit der Daten für Akteure im Gesundheitssystem; Nutzbarkeit der Daten im Hinblick auf die Reduzierung gesundheitlicher Ungleichheit; Nutzbarkeit der Daten für die Wissenschaft.

Zudem ist für ein Gesundheitsmonitoring eine Kontinuität in der Auswahl der Befragungsinhalte und -instrumente unverzichtbar, um Veränderungen über die Zeit aufzeigen zu können. 
Der Spezifik der einzelnen Monitoringkomponenten wird Rechnung getragen, indem jeweils zusätzliche Kriterien zur Reduzierung der Erhebungsinhalte auf ein für die Probanden vertretbares $\mathrm{Maß}$ angewendet werden.

\section{DEGS}

DEGS ist in der ersten Welle ein Untersuchungssurvey, in dem folgende Erhebungsinstrumente zum Einsatz kommen:

- computergestütztes ärztliches Inter-

view,

- Arzneimittelinterview,

- Selbstausfüll-Fragebögen,

- Laboruntersuchungen von Blut-, Urinproben,

- körperliche Untersuchungen.

Die unterschiedlichen Erhebungsmodi sind so eingesetzt und kombiniert, dass das Verhältnis zwischen Informationsgewinn und (zeitlicher) Belastung der Probanden optimiert wird. Dies bedeutet zum Beispiel, dass in die Fragebögen, die im Untersuchungszentrum eingesetzt werden, nur solche Fragen aufgenommen werden, die zur komplexen Auswertung der unterschiedlichen Informationen aus Interview, Untersuchungen und Laboranalysen unabdingbar sind. Fragen, die alleinstehend und unabhängig von anderen Informationen sind, also keine Vertiefung durch die zusätzlichen Erhebungskomponenten des Untersuchungssurveys erfahren können, werden in die reinen Befragungssurveys, also in GEDA verlagert.

Da DEGS eine Längsschnittkomponente durch die Einbeziehung der Probanden des BGS 98 enthält, war es naheliegend, die thematischen Schwerpunkte, aber auch die eingesetzten Instrumente des Surveys von 1998 einer kritischen Prüfung zu unterziehen, das hieß zu prüfen, inwieweit sie erneut in DEGS eingesetzt werden sollten. Diese Prüfung wurde vorgenommen und ein Teil der Untersuchungen und Fragen identisch übernommen. Andere wiederum wurden zugunsten einer neuen Schwerpunktsetzung in DEGS verändert oder ganz fallen gelassen. In Anbetracht des demografischen Wandels und dem sich verändernden Krankheitsspektrum in der Bevölkerung wurden zusätzlich zu den traditionellen Themen bestimmte Bereiche neu

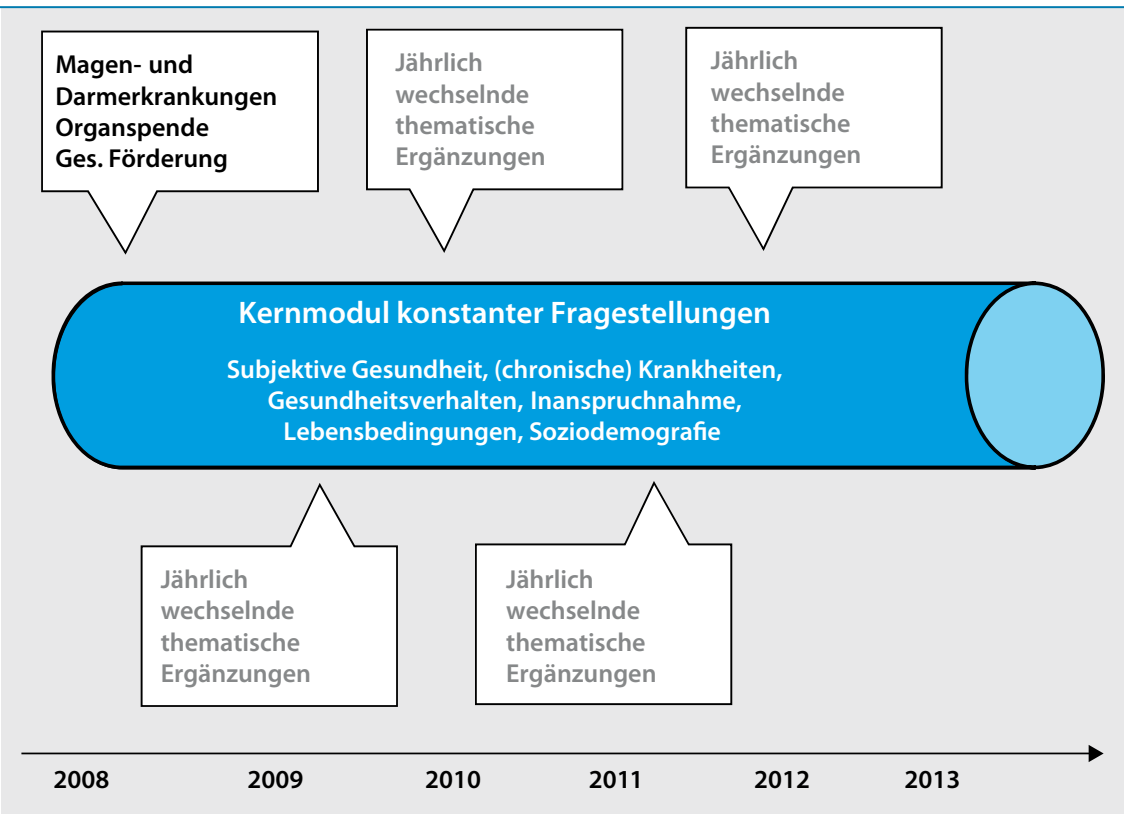

Abb. $4 \Delta$ Thematische Struktur von GEDA

beziehungsweise vertiefend in DEGS aufgenommen. Dies betrifft

Chronische Krankheiten (,Volkskrankheiten“): (undiagnostizierter) Diabetes mellitus; muskuloskelettale Erkrankungen; Herz-Kreislauf-Erkrankungen; Adipositas und metabolisches Syndrom.

Gesundheitliche Folgen des demografischen Wandels: Gesundheit der Bevölkerung über 65 Jahre, Multimorbidität, Gebrechlichkeit, Einschränkungen der körperlichen Funktionsfähigkeit und der kognitiven Leistungsfähigkeit.

Psychische Gesundheit: „Psychological Distress", Depressivität, Konsum von psychotropen Substanzen, Belastung durch chronischen Stress.

Eine Gesamtdarstellung findet sich in [20].

\section{GEDA}

Das Kernbefragungsset enthält Items aus den Bereichen Subjektive Gesundheit, (chronische) Krankheiten, psychische Gesundheit, Gesundheitsverhalten, Inanspruchnahme, gesundheitsbezogene Ressourcen und Belastungen, Lebensbedingungen und Soziodemografie. Die Bestimmung einer angemessenen themenspezifischen Periodik für Trendaussagen erfolgte im Vorfeld ebenso wie die Auswahl relevanter Gesundheitsdeterminanten, um tiefgehende Zusammenhangsa- nalysen der jährlichen Querschnittserhebungen zu ermöglichen. Das solchermaBen ermittelte Set von Kernfragestellungen weist gleichzeitig eine Schnittmenge zu DEGS auf, damit eine inhaltliche Vergleichbarkeit beider Erhebungen gewährleistet ist. Die in GEDA und DEGS übereinstimmend erfassten inhaltlichen Aspekte werden in DEGS durch Ergebnisse der medizinischen Untersuchungen, der Funktionstests und durch das ärztliche Interview erweitert und ermöglichen damit vertiefte, epidemiologische Auswertungen. Zusätzlich werden in GEDA, von Welle zu Welle wechselnd, weitere Themen einbezogen, die von aktueller Bedeutung sind oder für die es genügt, aufgrund relativ geringer Dynamik in größeren Zeitabständen Daten zu erheben (• Abb. 4). Diese zusätzlichen Inhalte werden von der Gesundheitspolitik und weiteren Akteuren des Gesundheitssystems vorgeschlagen oder sind von den Anforderungen des nationalen und europäischen Health Monitoring Systems bestimmt.

\section{KiGGS}

Die Inhalte von KiGGS-Welle 1 und die eingesetzten Instrumente haben bereits bei der Konzipierung der KIGGS-Basiserhebung einen mehrfachen, kriteriengestützten Auswahlprozess durchlaufen. 
In der ersten telefonischen Nachbefragung waren daher nicht so sehr neue Themen zu erschließen, sondern es wurden die eingesetzten Instrumente und Fragebögen der neuen Erhebungsart angepasst, die Themen reduziert, um die telefonischen Befragung nicht über maximal 45 Minuten andauern zu lassen.

Auf Basis der in KiGGS eingesetzten, altersgestaffelten (0-2, 3-6, 7-10, 11-13, 14-17 Jahre) Fragebögen für Eltern und Kinder und unter Berücksichtigung der Ergebnisse aus der KiGGS-Basiserhebung sowie den beiden methodischen Vorstudien erfolgte die Bearbeitung, Modifizierung und Adaptation der Fragebogeninstrumente an die Erfordernisse einer telefonischen Befragung. Aktuelle Literaturrecherchen, externe Expertisen und gesundheitspolitisch bedeutsame Fragestellungen wurden dabei explizit berücksichtigt. Ebenso wurden die einzelnen Themenbereiche nach den Kriterien: Vergleichbarkeit und Verknüpfbarkeit mit den Daten der Basiserhebung, Akzeptanz, Durchführbarkeit im Telefoninterview, Längsschnitttauglichkeit der Fragestellung und des Instruments, Differenzierungen des Instruments nach Adressat [Kind/Jugendliche(r)/junge(r) Erwachsene(r) bzw. Eltern] und Altersbereichen und Vermeidung von Redundanzen geprüft.

Die Kernbefragung umfasst alle wesentlichen Eckwerte zu den inhaltlich wichtigsten Themenbereichen des gesundheitlichen Geschehens: die gesundheitliche Lage einschließlich Schutz- und Risikofaktoren und Lebensqualität, akute und chronische Krankheiten, Schmerzen, psychische Auffälligkeiten und Krankheiten, Unfälle, Inanspruchnahme stationärer und ambulanter medizinischer Leistungen, Risikoverhalten, Lebensbedingungen und Sozialstatus. Die Kernkomponenten werden durch Zusatzmodule an Unterstichproben ergänzt. Diese können vertiefende Untersuchungen zu den schon in den Kernkomponenten einbezogenen Aspekten oder zu anderen gesundheitspolitischen Fragestellungen beinhalten. Es wird, ähnlich wie in der KiGGS-Querschnitts-Basiserhebung 2003-2006, zwei vertiefende Module mit den Schwerpunkten „Psychische Gesundheit“ sowie „Motorische Leistungsfähigkeit“ geben.

\section{Das Gesundheitsmonitoring des RKI im europäischen Kontext}

In der Konzeption des Gesundheitsmonitorings wurden von Anfang an die Anforderungen des sich im Aufbau befindlichen europäischen Health Monitoring Systems berücksichtigt. Dies betrifft die Vergleichbarkeit bzw. Kompatibilität

- des zugrunde gelegten konzeptionellen Modells von Gesundheit, - der einbezogenen Gesundheitsthemen, - der eingesetzten Erhebungsinstrumente,

- der erhobenen Daten.

Eines der Kernstücke des europäischen Monitoringsystems ist die Etablierung des europäischen Indikatorensystems ECHIM (European Community Health Indicators; http://www.echim.org). An der Konzeption und dem Aufbau dieses Indikatorensystems waren von deutscher Seite auch Wissenschaftler aus dem RKI aktiv beteiligt. Auf diese Weise wurde gewährleistet, dass die Erfahrungen aus den Gesundheitssurveys des RKI und aus der Gesundheitsberichterstattung des Bundes Eingang in dieses europäische Projekt fanden. Ziel des entwickelten Indikatorensets ist es, alle Aspekte der öffentlichen Gesundheit abzudecken, die Bedürfnisse der Nutzer, insbesondere der Gesundheitspolitik der Europäischen Kommission und der Mitgliedsstaaten zu berücksichtigen und künftigen Entwicklungs- und Handlungsbedarf im Gesundheitssektor aufzuzeigen.

Eine Durchsicht der in ECHIM vorgeschlagenen und für ein Gesundheitsmonitoring als essenziell angesehenen 88 Indikatoren zeigt, dass etwa die Hälfte davon nur mit Surveydaten oder unter Verwendung von Surveydaten gefüllt werden kann. Bei der inhaltlichen Konzeption von DEGS und GEDA wurden nicht nur die Thematiken dieser Indikatoren berücksichtigt, sondern soweit wie möglich auch die vorgeschlagenen Instrumente eingesetzt. Damit wurden die Grundvoraussetzungen für eine Vergleichbarkeit der Daten geschaffen; diese sind allerdings weiterhin nicht für alle europäischen Länder gewährleistet und werden auch durch kulturelle Besonderheiten in den einzelnen Mitgliedsstaaten, zum Bei- spiel hinsichtlich der Selbsteinschätzung des Gesundheitszustand oder der Bewertung des Gesundheitssystems erschwert.

\section{Kommunikation des \\ Gesundheitsmonitorings, Nutzung der erhobenen \\ Daten}

Am Beispiel des KiGGS soll stellvertretend für das Gesundheitsmonitoring insgesamt dargestellt werden, wie die Entwicklung der einzelnen Erhebungen transparent verläuft, wie die erhobenen Daten genutzt und die Ergebnisse in der Öffentlichkeit dargestellt werden:

Wie in [19] ausführlich beschrieben, wurde das Konzept für den Kinder- und Jugendgesundheitssurvey mehrfach extern begutachtet, in einer Pilotstudie getestet und das letztendliche Konzept für die Hauptstudie auf einem öffentlichen Symposium im Juni 2002 der interessierten Öffentlichkeit vorgestellt. Die Projektbeschreibung ist in [15] publiziert. Die Erhebung selbst wurde durch einen Wissenschaftlichen Beirat begleitet, die Feldarbeit war kontinuierlich einer externen Qualitätskontrolle unterzogen, im Epidemiologischen Bulletin des RKI wurde monatlich zu unterschiedlichen Aspekten des Surveys berichtet.

Erste Ergebnisse der Studie wurden bereits im September 2006 der Öffentlichkeit präsentiert, weitere Auswertungen folgten [25]. In den ersten zwei Jahren nach Beendigung der Feldarbeit wurden weit über 100 wissenschaftliche Publikationen von Mitarbeitern des RKI verfasst, zirka 50 Auswertungskooperationen mit wissenschaftlichen Einrichtungen abgeschlossen und eine nicht mehr überschaubare Zahl von Vorträgen zu KiGGS-Ergebnissen gehalten.

Seit Dezember 2009 sind die erhobenen Daten als Public Use File freigegeben und die Nachfrage wissenschaftlicher Einrichtungen nach diesem Datensatz übersteigt alle Erwartungen. Die Gesundheitswissenschaftler Deutschlands werden noch ein Vielfaches der bislang erlangten Ergebnisse zur Kinder- und Jugendgesundheit hinzufügen.

Der KiGGS hatte auch zum Ziel, sowohl für die Gesundheitsberichterstattung als auch für die Gesundheitspolitik 
dringend benötigte Daten zu erheben und damit Handlungsgrundlagen zu schaffen. So wurden auf der Grundlage von KiGGS-Daten diverse Berichte verfasst und Zuarbeiten geleistet zum 3. Armuts- und Reichtumsbericht der Bundesregierung (2008) [26], zum 11. Sportbericht der Bundesregierung [27], zum Gutachten des Sachverständigenrates im Gesundheitswesen „Lebensphasenspezifische Gesundheit von Kindern und Jugendlichen in Deutschland" [28] sowie zum 13. Kinder- und Jugendbericht [29]. Expertisen zur gesundheitlichen Ungleichheit bei Kindern und Jugendlichen in Deutschland sowie zur Gesundheit von Kindern und Jugendlichen mit Migrationshintergrund in Deutschland sind in der Reihe „Beiträge zur Gesundheitsberichterstattung" des RKI erschienen [30, 31]. Ausgehend von den durch die KiGGS-Ergebnisse erkannten Handlungsdefiziten wurden gemeinsam mit der Bundeszentrale für gesundheitliche Aufklärung $(\mathrm{BZgA})$ eine Bestandsaufnahme existierender gesundheitsfördernder Maßnahmen und Präventionsansätze vorgenommen und Handlungsempfehlungen abgeleitet [32]. Dass die Bundesregierung ihre Strategie zur Kindergesundheit [33] ebenso auf KiGGS-Ergebnisse aufbaut wie die BMGInitiative „Leben hat Gewicht“ [34] ist der Beweis dafür, dass diese Surveyerhebung ihrem Anspruch, „Daten für Taten“ zu schaffen, gerecht geworden ist.

Analog zum Modell „KiGGS“ wird auch mit den beiden anderen Monitoringkomponenten verfahren: Die Projektbeschreibung, die für DEGS entwickelt wurde, hat ebenfalls eine externe Begutachtung durchlaufen, wird in [20] publiziert und wurde auf einem öffentlichen Symposium im September 2008 vorgestellt. Dieses Symposium war der Auftakt für eine geplante Folge von Monitoringsymposien, auf denen jeweils die aktuellen Erhebungen vorgestellt und diskutiert werden. Für September 2009 ist ein Symposium zu GEDA geplant.

Um eine wissenschaftliche Begleitung des Gesundheitsmonitorings zu gewährleisten, wurde im Dezember 2008 eine Expertenkommission für „Gesundheitsberichterstattung und Gesundheitsmonitoring" an das RKI berufen (siehe http:// www.rki.de). Ebenso wie für KiGGS wird wiederum über GEDA und DEGS im Epidemiologischen Bulletin des RKI informiert $[35,36,37,38]$.

Die Publikationsstrategien für DEGS werden analog zu der bewährten KiGGS-Strategie entwickelt. Für die jährlich durchgeführten telefonischen Befragungen GEDA ergeben sich zusätzlich andere Möglichkeiten. Eine schnelle Nutzbarmachung und Publikation der Daten ist über ein gestuftes Vorgehen geplant: Möglichst zeitnah nach Datenaufbereitung soll jährlich ein indikatorenbasierter Ergebnisbericht publiziert werden. Schwerpunkt des Berichts sollen Informationsblätter zu den wichtigsten Indikatoren sein, in denen standardisiert die Public-Health-Relevanz der jeweiligen Kennzahl sowie deren Berechnung beschrieben und ein Grundauswertungstableau dargestellt wird, regionale Vergleiche durchgeführt werden und eine Bewertung der Ergebnisse vorgenommen wird. Zusätzlich soll ein komprimierter Datensatz in das Internet gestellt werden, der es den Nutzern ermöglicht, eigene Tabellen unter bestimmten Gliederungsmerkmalen abzurufen. Eine detaillierte Darstellung und Aufbereitung der Indikatoren ist im Rahmen der Entwicklung eines Bundesindikatorensystems der Gesundheitsberichterstattung des Bundes vorgesehen. In einem weiteren Schritt sollen die Daten für die GBE-Publikationen genutzt und schließlich vertiefte epidemiologische Analysen in Fachzeitschriften publiziert werden.

Sämtliche Daten, die im Rahmen des Monitoringsystems erhoben werden, sollen, wie bisher bei allen Gesundheitssurveys, spätestens zwei Jahre nach Beendigung der Feldarbeit in Form eines dokumentierten Public Use Files freigegeben werden. Bereits vorher sind Daten im Rahmen einer Kooperationsvereinbarung zu erhalten.

\section{Weiterentwicklung des Gesund- heitsmonitorings/Perspektiven}

Das hier skizzierte Gesundheitsmonitoring-System darf und kann kein starres, einmal festgelegtes System sein. Neue gesundheitliche Herausforderungen, Fortentwicklungen in Prävention und Versorgung, gesundheitspolitische Anforde- rungen, weitere Bestrebungen zu Qualität und Wirtschaftlichkeit im Gesundheitswesen, innovative Methoden der Datenerhebung und -Weiterleitung erfordern die regelmäßige Analyse der bestehenden Inhalte und Methoden sowie entsprechende Anpassungen der Gesundheitssurveys und des Gesamtsystems. Beispielhaft sollen für die Aspekte „Inhalte“, „Instrumente “, „Methoden" und „Vernetzung von Daten" Entwicklungsmöglichkeiten aufgezeigt werden.

Inhalte. Zur Zunahme des Anteils an psychischen Erkrankungen in der Bevölkerung, die sich beispielsweise in den Krankschreibungen und gesundheitsbedingten Frühberentungen niederschlägt, liegen seit 1998 keine aktuellen, bevölkerungsrepräsentativen Daten vor. Die vorhandenen Prozessdaten lassen keinen oder nur einen eingeschränkten Schluss auf die Lebensbedingungen, Folgeerkrankungen und unterstützende sowie Risikofaktoren zu. Aus diesem Grund wurden in den DEGS im allgemeinen Befragungsteil verschiedene Instrumente zur Erfassung von psychischem Distress, Depressivität und chronischem Stress sowie die Erfassung ärztlich diagnostizierter psychischer Krankheiten im ärztlichen Interview integriert. Die Vertiefung dieser Fragestellungen sowie die Erfassung der Versorgungssituation in einem gesonderten Modul zur psychischen Gesundheit sind vorgesehen. Die Häufigkeit und die Wahrnehmung der gesellschaftlichen Bedeutung bestimmter Krankheitsbilder verändert sich indes stetig: Durch die $\mathrm{Zu}-$ nahme des Anteils der Älteren in der Bevölkerung wird möglicherweise auch die Zahl der Menschen ansteigen, die in ihren Alltagsfunktionen eingeschränkt sind. Entscheidend für die Teilhabe am gesellschaftlichen Leben ist aber weniger das Vorhandensein einer Einschränkung als das Vorliegen oder Fehlen einer adäquaten Unterstützung. Die Entwicklung eines „Disability-and Social Integration Modules", das diese Aspekte einbezieht und sich an der ICF, der internationalen Klassifikation funktioneller Einschränkungen orientiert, vollzieht sich bereits auf europäischer Ebene [39]. Ansätze in diese Richtung wurden in den Fragebogen für die über 65-Jährigen im DEGS 
integriert; gerade unter dem Aspekt von Verlaufsuntersuchungen und der Einbeziehung hochaltriger Menschen wird diese Thematik wahrscheinlich in der nächsten Welle noch ausgebaut werden.

Instrumente. Der steigende Anteil an älteren Probanden in den Befragungs- und Untersuchungssurveys macht es auch erforderlich, Instrumente einzusetzen, die eine Bewertung der kognitiven Fähigkeiten der Probanden ermöglichen. Im DEGS wird bereits bei den über 65-jährigen Probanden ein Zahlen-SymbolTest eingesetzt, der verschiedene Dimensionen der kognitiven Leistungsfähigkeit erfasst. In einer Pilotstudie im Rahmen des BMBF-geförderten Projekts OMAHA (Operationalisierung von Multimorbidität und Autonomie im höheren Alter) [40] werden Tests zur zeitlichen Orientierung der Probanden, zur verbalen Intelligenz und zum Gedächtnis am Telefon erprobt. Damit kann das Auftreten kognitiver Störungen gemessen werden. In der weiteren Entwicklung der Gesundheitssurveys müssen daher zunehmend Instrumente zur Messung spezifischer Störungen für verschiedene Erhebungsformen (face-to-face, postalisch-schriftlich, telefonisch, via Internet) angepasst und überprüft werden.

Methoden. Die fortwährende Weiterentwicklung der Kommunikationstechnologie und die damit einhergehenden bevorzugten Kommunikationswege der Bürgerinnen und Bürger müssen auch im Design von Gesundheitssurveys berücksichtigt werden. Die zunehmende Verbreitung des Internets in Haushalten [41] eröffnet neue und kostengünstige Erhebungswege. Voraussetzung ist allerdings die Lösung noch bestehender Probleme bezüglich der Gewinnung einer bevölkerungsrepräsentativen, nicht geklumpten Stichprobe teilnahmewilliger Probandinnen und Probanden. Denkbar sind auch Erhebungsverfahren, bei denen einer vorher gezogenen repräsentativen Stichprobe verschiedene Möglichkeiten der Befragung angeboten werden: zum Beispiel telefonisch, schriftlich-postalisch oder über das Internet. Hierfür ist erforderlich, in vorangehenden Methodenstudien den Einfluss der jeweiligen Erhebungsme- thode auf das Antwortverhalten zu analysieren. Eine weitere Möglichkeit stellt die Verwendung von Daten aus elektronischen Patientenakten dar. Schließlich müssen spezifische Zugangswege für Personen in Institutionen oder für andere schwer erreichbare Bevölkerungsgruppen entwickelt werden. Eine besondere Herausforderung wird es auch sein, schwer erreichbare oder besonders mobile Probanden aus Kohortenstudien möglichst langfristig durch entsprechende Methoden in die Studien einzubinden.

Vernetzung von Daten. In einer Analyse zu den Einflussfaktoren der ambulanten Versorgung in Deutschland wurden die Daten aus dem Bundes-Gesundheitssurvey 1998 um regionale Strukturdaten und aggregierte Daten aus der vertragsärztlichen Abrechnung ergänzt. Damit wurde es möglich, das Inanspruchnahmeverhalten der Surveyteilnehmer vor dem Hintergrund der Struktur des ärztlichen Angebots sowie weitere Merkmale des jeweiligen Kreises zu interpretieren [42]. Künftig könnten Surveydaten noch mit weiteren kleinräumigen Daten verknüpft werden, zum Beispiel könnten für die Analyse von Unfällen und des Unfallgeschehens Daten aus KiGGS mit Daten aus dem Kinderunfallatlas der Bundesanstalt für Straßenwesen [43] verbunden werden. Perspektivisch ist auch denkbar, auf Personenebene Daten aus Surveys mit Daten aus der ärztlichen Versorgung zu verknüpfen. In Fortsetzung eines vom BMBF geförderten Verbundprojektes zur Sicherung der hausärztlichen Versorgungsqualität [44] wurden am RKI die Möglichkeiten der Nutzbarkeit routinemäßig erhobener, elektronischer Daten in der Hausarztpraxis für die Begleit- und Evaluationsforschung überprüft. Künftig wäre auch der umgekehrte Weg denkbar, bei dem Befragungs- und Untersuchungsdaten der Surveyprobanden gezielt um elektronisch verfügbare Daten derselben Studienteilnehmer aus der ambulanten Versorgung ergänzt werden, vorausgesetzt, die Einwilligung der Probanden liegt vor, die datenschutzrechtlichen Auflagen lassen sich erfüllen und die jeweiligen Ärzte lassen sich zur Teilnahme gewinnen.
Fazit

Das hier vorgestellte Gesundheitsmonitoring am RKI soll als ein flexibel gestaltetes und an künftige Anforderungen adaptierbares, nachhaltiges System gewährleisten, dass der Gesundheitsberichterstattung und der Gesundheitspolitik jederzeit umfangreiche Informationen zur Gesundheit, zum Gesundheitsverhalten und zur gesundheitlichen Versorgung der in Deutschland lebenden Bevölkerung zur Verfügung stehen, dass Deutschland seinen Verpflichtungen zur Lieferung von Gesundheitsdaten an die Europäische Gemeinschaft nachkommen kann, dass mithilfe der Monitoringdaten wissenschaftlichen Fragestellungen nachgegangen, gesundheitspolitische Maßnahmen evaluiert und Auswirkungen festgestellt werden können. Damit soll das Gesundheitsmonitoring am RKI einen Beitrag zur Erfüllung der eingangs zitierten Hauptaufgaben des Instituts - „Erkennen, Bewerten, Handeln“ - leisten und letztendlich der Gesunderhaltung der Bevölkerung dienen.

\section{Korrespondenzadresse}

\section{Dr. B.-M. Kurth}

Abteilung für Epidemiologie und Gesundheitsberichterstattung,

Robert Koch-Institut

Postfach 650261, 13302 Berlin

KurthB@rki.de

\section{Literatur}

1. Bundesministerium für Gesundheit (2007) Das Robert Koch-Institut - das Public Health Institut für Deutschland. Bundesministerium für Gesundheit, Referat Öffentlichkeitsarbeit, Berlin

2. Kurth BM, Ziese T (2005) Gesundheitssurveys des Robert Koch-Instituts. Asgard-Verlag Dr. Werner Hippe GmbH, Sankt Augustin

3. Bundesgesundheitsbl Gesundheitsforsch Gesundheitsschutz (2003) Gesundheitsziele haben die Erhaltung oder Verbesserung von Gesundheit zum Ziel. Bundesgesundheitsbl Gesundheitsforsch Gesundheitsschutz 46:103-155

4. Robert Koch-Institut (2000) Schutzimpfungen. Gesundheitsberichterstattung des Bundes, Heft 1. Eigenverlag, Berlin (Aktualisierte Neuauflage im Jahr 2004)

5. Robert Koch-Institut (2002) Chronische Schmerzen - Kopf- und Rückenschmerzen, Tumorschmerzen. Gesundheitsberichterstattung des Bundes Heft 7. Eigenverlag, Berlin

6. Robert Koch-Institut (2004) Selbsthilfe im Gesundheitsbereich. Gesundheitsberichterstattung des Bundes, Heft 23. Eigenverlag, Berlin 
7. Robert Koch-Institut (2005) Diabetes mellitus. Gesundheitsberichterstattung des Bundes, Heft 24. Eigenverlag, Berlin

8. Robert Koch-Institut (2005) Brustkrebs. Gesundheitsberichterstattung des Bundes, Heft 25. Eigenverlag, Berlin

9. Robert Koch-Institut (Hrsg) (2006) Bürger- und Patientenorientierung. Gesundheitsberichterstattung des Bundes, Heft 32. Eigenverlag, Berlin

10. Robert Koch-Institut (2005) Armut, soziale Ungleichheit und Gesundheit. Expertise des Robert Koch-Instituts zum 2. Armuts- und Reichtumsbericht der Bundesregierung. Beiträge zur Gesundheitsberichterstattung des Bundes. Eigenverlag, Berlin

11. Bundesministerium für Ernährung, Landwirtschaft und Verbraucherschutz, Bundesministerium für Gesundheit (2008) IN FORM. Deutschlands Initiative für gesunde Ernährung und mehr Bewegung. Der Nationale Aktionsplan zur Prävention von Fehlernährung, Bewegungsmangel, Übergewicht und damit zusammenhängenden Krankheiten http:// www.bmg.bund.de/cln_110/nn_1168248/SharedDocs/Standardartikel/DE/AZ/L/Glossarbegriff-Leben-hat-Gewicht.html (Stand: 13.2.2009)

12. Kreuter H, Klaes L, Hoffmeister H, Laaser U (1995) In: Prävention von Herz-Kreislaufkrankheiten. Ergebnisse und Konsequenzen der Deutschen HerzKreislauf-Präventionsstudie. Juventa, Weinheim

13. Bellach BM, Knopf H, Thefeld W (1998) Der Bundes-Gesundheitssurvey 1997/98. Gesundheitswesen 60 (Sonderheft 2):S59-S68

14. Das Gesundheitswesen (1999) Der Bundes-Gesundheitssurvey 1998 - Erfahrungen, Ergebnisse, Perspektiven. Gesundheitswesen 61 (Sonderheft 2)

15. Das Gesundheitswesen (2002) Kinder- und Jugendgesundheitssurvey - Konzept, Ziele, Inhalte, Instrumente, Pretest. Gesundheitswesen 64 (Sonderheft 1)

16. Kurth BM (2006) Epidemiologie und Gesundheitspolitik. Bundesgesundheitsbl Gesundheitsforsch Gesundheitsschutz 49(7):637-647

17. Kurth BM, Ziese T, Tiemann F (2005) Gesundheitsmonitoring auf Bundesebene. Ansätze und Perspektiven. Bundesgesundheitsbl Gesundheitsforsch Gesundheitsschutz 48:261-272

18. Kurth BM (2008) Zum aktuellen Stand eines Gesundheitsmonitoringssystems am Robert Koch-Institut. In: Statistik und Wissenschaft. Datengrundlagen für die Gesundheitsforschung in Deutschland. Statistisches Bundesamt (Hrsg) Band 9:34-36

19. Kurth BM (2007) Der Kinder- und Jugendgesundheitssurvey (KiGGS): Ein Überblick über Planung, Durchführung und Ergebnisse unter Berücksichtigung von Aspekten eine Qualitätsmanagements. Bundesgesundheitsbl Gesundheitsforsch Gesundheitsschutz 50 (Heft 5/6):533-546

20. Robert Koch-Institut (2009) DEGS: Studie zur Gesundheit Erwachsener in Deutschland - Projektbeschreibung. Beiträge zur Gesundheitsberichterstattung des Bundes. Eigenverlag, Berlin (in Vorbereitung)

21. Robert Koch-Institut (2009) Information zu DEGS: Einladung und Probandengewinnung. Epid Bull 6:315-317

22. Robert Koch-Institut (2005) Erster telefonischer Gesundheitssurvey des Robert Koch-Instituts Methodische Beiträge. Beiträge zur Gesundheitsberichterstattung des Bundes. Eigenverlag, Berlin

23. Gabler S, Häder S (2002) Telefonstichproben. Methodische Innovationen und Anwendungen in Deutschland. Waxmann, Münster
24. Link MW, Mokdad A (2005) Advance letters as a means of improving respondents cooperation in random digit dial studies. Public Opinion Quarterly 69:572-587

25. Bundesgesundheitsbl Gesundheitsforsch Gesundheitsschutz (2007) Ergebnisse des Kinder- und Jugendgesundheitssurveys (KiGGS). Bundesgesundheitsbl Gesundheitsforsch Gesundheitsschutz 50 (Heft 5/6)

26. Bundesregierung (2008) Lebenslagen in Deutschland - Der 3. Armuts- und Reichtumsbericht der Bundesregierung. Drucksache 16/9915

27. Bundesregierung (2006) 11. Sportbericht der Bundesregierung. Drucksache 16/3750

28. Robert Koch-Institut (2008) Beiträge zur Gesundheitsberichterstattung des Bundes. Lebensphasenspezifische Gesundheit von Kindern und Jugendlichen in Deutschland. Eigenverlag, Berlin

29. Bundesregierung (2008) 13. Kinder- und Jugendbericht - Bericht über die Lebenssituation junger Menschen und die Leistungen der Kinder- und Jugendhilfe in Deutschland. Eigenverlag, Berlin

30. Robert Koch-Institut (2008) Gesundheitliche Ungleichheit bei Kindern und Jugendlichen in Deutschland. Eigenverlag, Berlin

31. Robert Koch-Institut (2008) Beiträge zur Gesundheitsberichterstattung des Bundes. Kinder- und Jugendgesundheitssurvey (KiGGS) 2003-2006: Kinder und Jugendliche mit Migrationshintergrund in Deutschland. Eigenverlag, Berlin

32. Robert Koch-Institut und Bundeszentrale für gesundheitliche Aufklärung (2008) Erkennen - Bewerten - Handeln: Zur Gesundheit von Kindern und Jugendlichen in Deutschland. RKI und BZgA, Eigenverlag, Berlin

33. Bundesministerium für Gesundheit (2008) Strategie der Bundesregierung zur Förderung der Kindergesundheit http://www.bmg.bund.de/cln_ 110/nn_1168720/SharedDocs/Standardartikel/ DE/AZ/K/Glossarbegriff-Kindergesundheit.html (Stand:13.2.2009)

34. Bundesministerium für Gesundheit (2007) Initiative "Leben hat Gewicht - gemeinsam gegen den Schlankheitswahn" http://www.leben-hat-gewicht.de/cln_117/sid_5A5FA75AEB1AB6A0C69EEF173CF95D95/DE/Home/home node.html?_nnn=true (Stand:13.2.2009)

35. Robert Koch-Institut (2008) Gesundheit in Deutschland aktuell: Feldbeginn des telefonischen Gesundheitssurveys 2008/2009 am RKI. Epid Bull 37:315-317

36. Robert Koch-Institut (2008) DEGS: Die neue bundesweite Studie zur Gesundheit Erwachsener als Komponente des Gesundheitsmonitorings des Robert Koch-Instituts. Epid Bull 41:353-355

37. Robert Koch-Institut (2008) DEGS - RKI hat jetzt mit der Feldarbeit begonnen. Epid Bull 49:440 443

38. Robert Koch-Institut (2009) Information zu DEGS: Einladung und Probandengewinnung. Epid Bull 6:315-317

39. Rat der Europäischen Union (2007) Mitteilung der Kommission an den Rat, das Europäische Parlament, den Europäischen Wirtschafts- und Sozialausschuss und den Ausschuss der Regionen. Situation von Menschen mit Behinderungen in der Europäischen Union: Europäischer Aktionsplan 2008-2009. Brüssel KOM (2007) 738 endgültig

40. OMAHA - Multimorbidität im höheren Lebensalter. http://www.rki.de/cln_091/nn_204976/DE/ Content/GBE/Forschungsvorhaben/Morbiditaetsspektrum/omaha/omaha_node.html?_nnn=true (Stand:13.2.09)
41. Initiative D 21 (2008) (N)onliner Atlas. Eine Topographie des digitalen Grabens durch Deutschland. TNS Infratest Holding

42. Thode M, Bergmann E, Kamtsiuris P, Kurth BM (2005) Einflussfaktoren auf die ambulante Inanspruchnahme in Deutschland. Bundesgesundheitsbl Gesundheitsforsch Gesundheitsschutz 48(3):296-306

43. http://www.bast.de/nn_42254/DE/Publikationen/ Berichte/unterreihe-m/2008-2009/m192.html (Stand:13.2.2009)

44. http://www.rki.de/cln_091/nn_204824/DE/Content/GBE/Forschungsvorhaben/Versorgung/Evaluationsforschung/Evaluationsforschung_node.html?_nnn=true (Stand:13.2.2009) 


\section{S. Förderreuther, A. Straube Kopfschmerzen} Reihe Klinische Neurologie

W. Kohlhammer Verlag 2009, 1. Aufl., 264 S., (ISBN 3-17-018461-9), brosch., 49.90 EUR

Kopfschmerzen sind eines der häufigsten Symptome, die Patienten zum Arzt führen, und Patienten mit chronischen Kopfschmerzen stellen einen großen Anteil in der Praxis des Schmerztherapeuten dar. Das Wissen um Diagnose und Therapie der verschiedenen Kopfschmerzformen ist jedoch bei vielen Ärzten, und selbst bei vielen Schmerztherapeuten noch nicht ausreichend vorhanden. Die Therapie der chronischen Kopfschmerzen unterscheidet sich deutlich von der Behandlung anderer chronischer Schmerzsyndrome, und weist auch zwischen den mehr als 300 verschiedenen Kopfschmerzformen deutliche Unterschiede auf.

In dem neuen Buch „Kopfschmerzen“ in der Reihe zur klinischen Neurologie es Kohlhammer Verlages stellen die Autoren die Diagnostik und Therapie der häufigsten und relevanten Kopfschmerzformen übersichtlich dar. Das Buch berücksichtigt die aktuellen Entwicklungen nicht nur bei der medikamentösen Therapie, sondern in allen Bereichen der Behandlung.

Im einleitenden Kapitel wird die Klassifikation der Kopfschmerzen nach der Internationalen Kopfschmerzgesellschaft dargestellt. In der überarbeiteten Version von 2004 werden die Fortschritte im pathophysiologischen Verständnis und die verbesserten Beschreibungen des klinischen Bildes der einzelnen Kopfschmerzarten aufgenommen. Es folgt eine ausführliche Darstellung von Anamnese, körperlicher Untersuchung und apparativer Diagnostik.

Vor der Abhandlung der einzelnen Kopfschmerzarten steht ein Kapitel zu Notfällen, bei denen die Kopfschmerzen Symptom einer potentiell lebensbedrohlichen Erkrankung sind oder bei ausbleibender Behandlung zu bleibenden Schäden führen können. Die kurze, übersichtliche Darstellung mit den wichtigsten Warnzeichen (Red Flags) hilft in der klinischen Praxis, diese Notfälle rechtzeitig zu erkennen.

Die Behandlung der verschiedenen Migräneformen, sowohl im Anfall wie in der Prophyla$\mathrm{xe}$, wird ausführlich dargestellt. Interessant ist die ausführliche Übersicht zur Pathophysiolo- gie. In der Behandlung wird die Evidenz aus der Literatur mit praktischen Hinweisen für die klinische Praxis kombiniert.

Bei den sekundären Kopfschmerzen ist besonders zu begrüssen, dass die medikamenteninduzierten Kopfschmerzen ausführlich dargestellt werden. Leider ist das Wissen um diese häufig auftretende Kopfschmerzform noch nicht weit genug verbreitet, und auch Schmerztherapeuten fördern durch den unkritischen Einsatz von Analgetika mitunter die Entstehung solcher Schmerzsyndrome. Insgesamt bietet das Buch eine gute und aktuelle Übersicht über die häufigen und wichtigen Kopfschmerzformen. Die beiliegende CD bietet weiteres Material, z.B. zur Pharmakologie der wichtigsten Analgetika und eine Aufstellung der weiterführenden Fachliteratur. Dieses kleine Buch wird den Ärzten helfen, die in der Arztpraxis oder im Krankenhaus immer wieder Kopfschmerzpatienten begegnen, und auch dem Schmerztherapeuten, der außerhalb der Neurologie in spezialisierten oder nichtspezialisierten Einrichtungen arbeitet. Das Buch ist gut geeignet für Kitteltasche oder Schreibtisch, und ich hoffe, dass mit diesem Buch die Behandlung für viele Kopfschmerzpatienten in Deutschland verbessert werden wird.

L. Radbruch (Aachen)

\section{R. Schwarz; S. Singer \\ Einführung Psychosoziale Onkologie}

Reinhardt UTB 2008, 314 S., 39 Abb., 49 Tab., 26.00 EUR

Weil sich eine Krebserkrankung auf alle Bereiche der körperlichen, seelischen und sozialen Existenz auswirkt, braucht die Psychosoziale Onkologie einen bio-psychosozialen Verständnisansatz, als Grundlage für eine wissenschaftlich fundierte und fachlich kompetente Begleitung, Beratung und Behandlung von Tumorpatienten und ihren Angehörigen. Mit der „Einführung Psychosoziale Onkologie" liegt erstmals ein ebenso umfassendes wie kompaktes Lehrbuch vor, in dem die Autoren eine Landkarte zur Orientierung in diesem vielschichtigen Fachgebiet zur Verfügung stellen und sie mit praxisrelevantem Erkenntnisgewinn verbinden. Das Buch vermittelt eingehende Kenntnisse über typische psychosoziale Problemlagen von Tumorpatienten, über Bewältigungs- und Verarbeitungsprozesse und spezielle diagnostische Methoden. Es informiert über die vielfältigen Ansätze von psychosozialen Interventionen, wobei auch Methoden mit unbewiesener Wirksamkeit kritisch diskutiert werden. Aktuelle Forschungsergebnisse, etwa zum Stellenwert psychosozialer Faktoren bei Entstehung und Verlauf von Krebserkrankungen werden da einbezogen, wo sie für das Verständnis der Patientenperspektive oder für die psychosoziale Versorgung Bedeutung haben. An Beispielen wie Tumorschmerz, Fatigue, Beeinträchtigungen von Sexualität werden spezifische Auswirkungen von Krebserkrankung und Behandlung auf das körperliche und psychische Erleben, und auf soziale Rollen und Funktionen veranschaulicht, wobei soziokulturelle Aspekte immer mitberücksichtigt werden. Der Überblick über psychosoziale Versorgungsmodelle, psychoonkologische Fachverbände und Fortbildungsangebote, im Besonderen zur Weiterbildung Psychosoziale Onkologie (WPO) der PSO/DKG und dapo, verdeutlicht den Grad an Professionalisierung, den das Fachgebiet erreicht hat. Wer Tumorpatienten im klinischen oder forschenden Kontext betreut, sei es als Ärztin, Pflegende, Sozialarbeiter oder Psychologe, profitiert von dieser fundierten, vielseitigen und anschaulichen Einführung in die zentralen Themen der Psychosozialen Onkologie mit Kenntnisgewinn und persönlichem Nutzen. Mit diesem Buch, dem viele Leser zu wünschen sind, tragen die Autoren dazu bei, die psychosoziale Unterstützung von Tumorpatienten weiter zu verbessern.

\section{Monika Keller (Heidelberg)}

